\title{
Meta-Analysis of Visual Evoked Potential and Parkinson's Disease
}

\author{
Song-bin He $\mathbb{D}^{1},{ }^{1}$ Chun-yan Liu, ${ }^{2}$ Lin-di Chen, ${ }^{1}$ Zhi-nan Ye, ${ }^{3}$ Ya-ping Zhang, \\ Wei-guo Tang, ${ }^{1}$ Bin-da Wang, ${ }^{1}$ and Xiang Gao ${ }^{4}{ }^{4}$ \\ ${ }^{1}$ Department of Neurology, Zhoushan Hospital, Wenzhou Medical University, Zhoushan 316021, China \\ ${ }^{2}$ Department of Critical Care Medicine, Huzhou Central Hospital, Huzhou 313000, China \\ ${ }^{3}$ Department of Neurology, Taizhou Municipal Hospital, Taizhou 318000, China \\ ${ }^{4}$ Department of Nutritional Sciences, The Pennsylvania State University, USA \\ Correspondence should be addressed to Xiang Gao; xxg14@psu.edu
}

Received 21 February 2018; Revised 20 May 2018; Accepted 3 June 2018; Published 11 July 2018

Academic Editor: Antonio Pisani

Copyright (C) 2018 Song-bin He et al. This is an open access article distributed under the Creative Commons Attribution License, which permits unrestricted use, distribution, and reproduction in any medium, provided the original work is properly cited.

Background. Previous studies suggested that visual evoked potential (VEP) was impaired in patients with Parkinson's disease (PD), but the results were inconsistent. Methods. We conducted a systematic review and meta-analysis to explore whether the VEP was significantly different between PD patients and healthy controls. Case-control studies of PD were selected through an electronic search of the databases PubMed, Embase, and the Cochrane Central Register of Controlled Trials. We calculated the pooled weighted mean differences (WMDs) and 95\% confidence intervals (CIs) between individuals with PD and controls using the random-effects model. Results. Twenty case-control studies which met our inclusion criteria were included in the final metaanalysis. We found that the P100 latency in PD was significantly higher compared with healthy controls (pooled WMD $=6.04,95 \%$ CI: 2.73 to $9.35, P=0.0003, n=20$ ). However, the difference in the mean amplitude of $P 100$ was not significant between the two groups (pooled $\mathrm{WMD}=0.64,95 \% \mathrm{CI}:-0.06$ to $1.33, P=0.07$ ) based on 10 studies with the P100 amplitude values available. Conclusions. The higher P100 latency of VEP was observed in PD patients, relative to healthy controls. Our findings suggest that electrophysiological changes and functional defect in the visual pathway of PD patients are important to our understanding of the pathophysiology of visual involvement in PD.

\section{Background}

Parkinson's disease (PD) is one of the most common neurodegenerative disorders in the world. The prevalence of $\mathrm{PD}$ is expected to rise steadily in the future as the human population ages [1]. Visual dysfunction is a common nonmotor symptom in individuals with $\mathrm{PD}$, including abnormal contrast sensitivity, motion perception abnormalities, and impaired visual acuity and color vision $[2,3]$. Visual dysfunction that occurs in PD is subtle and could be easily demonstrated through electrophysiologic testing, such as the visual evoked potential (VEP). VEP is a potential change recorded in the visual cortex after retinal received light stimulation, which reflects the functional status of the entire visual pathway. VEP latency is less likely to be affected by dopaminergic drugs and seems to be a more sensitive measure of foveal electrical activity than VEP amplitude. It is thought that abnormal latency is due to delayed conduction in visual pathways affected by the process of demyelination and/or plaque formation [2]. The P100 latency of VEP is usually used to determine the abnormalities of the visual pathway due to the relatively small individual difference.

As the pathological hallmark of PD is progressive loss of dopaminergic neurons in the substantia nigra, the human retina also contains dopaminergic amacrine and interplexiform cells, which play a regulatory role. Several observations support the concept that dopamine has a specific function in the retina of primates $[4,5]$. The chemical protoxin MPTP (1-methyl-4phenyl-1-2-3-6-tetrahydropyridine), which produces a clinical parkinsonian syndrome, significantly lowers retinal dopamine. Similarly, intravitreal injection of the neurotoxin 6-hydroxydopamine into aphakic monkeys resulted in abnormalities in both the phase and amplitude of the pattern VEP [6].

Visual dysfunction was observed in some early PD patients not yet undergoing L-dopa therapy, indicating that 
visual deficiency could be one of the prodromal symptoms of $\mathrm{PD}[3,7,8]$. However, previous studies regarding visual dysfunction, as assessed by the VEP, in PD patients versus general populations generated mixed results [8-10], which could be due to small sample sizes of these individual studies (PD case numbers $<50$ for all studies). We thus performed a systematic review and meta-analysis to comprehensively assess whether the pattern reversal VEP latency, as the primary exposure, was different between PD patients and controls. However, we also examined other visual indices, including pattern reversal VEP amplitude, intraocular difference of P100 latency, and N75 latency.

\section{Materials and Methods}

This meta-analysis was performed according to the Preferred Reporting Items for Systematic Reviews and Meta-Analyses (PRISMA) statement (PRISMA Checklist in supplementary materials (available here)) [11].

2.1. Search Strategy, Study Inclusion Criteria, and Data Extraction. Two of the coauthors (Chun-yan Liu and Ya-ping Zhang) independently searched the literature and extracted the relevant information from the eligible studies. A systematic review of the literature was conducted using the databases PubMed, Embase, and the Cochrane Central Register of Controlled Trials from 1 January 1978 up to 10 May 2016 to identify the relevant studies. We searched the medical subject heading $(\mathrm{MeSH})$ terms "Parkinson's disease" and "visual evoked potentials" in PubMed, respectively, and found out their entry terms. We only included English papers. Retrieved studies were imported into Mendeley Desktop (version 1.16; PDFTron ${ }^{\mathrm{TM}}$ Systems Inc.), where duplicate articles were deleted. Titles and abstracts of the remaining studies were independently scanned by Chun-yan Liu and Ya-ping Zhang. The full texts of the potentially relevant reports were then read to determine whether they met the inclusion criteria. The reference lists from all included studies were also examined.

Studies fulfilling the following inclusion criteria were included in the present meta-analysis: (1) participants were adult; (2) study design was a case-control study including a group of diagnosed idiopathic PD; (3) all participants underwent pattern reversal VEP examination, and both visual acuity test and ophthalmologic evaluation showed normal results; (4) information on peak latency of the P100 component was provided; and (5) sample size was greater than 10 in each group. Exclusion criteria were as follows: (1) participants were PD patients with dementia or undergoing deep brain stimulation; (2) authors did not make pattern reversal VEP examination; (3) studies without healthy control group; (4) studies did not provide the P100 data of pattern reversal VEP; (5) review papers; (6) reports published only in the abstract form; and (7) papers were not written in English.

A standardized data extraction form was used to collect relevant information including the name of the first author, publication year, study country, PD diagnosis criteria, mean disease duration, scales used for evaluating motor and cognitive function, number of eyes, mean age, sex, and latency of P100.

Two reviewers (Chun-yan Liu and Ya-ping Zhang) separately evaluated studies, and discrepancies were resolved by discussion. If disagreements existed, in very few cases, two reviewers consulted a third party (e.g., Xiang Gao) until both reviewers reached an agreement.

2.2. Study Quality Assessment. We assessed the methodologic quality of included studies based on the NewcastleOttawa Scale (NOS) [12] for quality of the case-control study. The NOS uses a star rating system to judge the quality based on 3 dimensions of the study: selection, comparability, and exposure. A study could be scored a maximum of one star for each item numbered within the categories of selection and exposure, while at most two stars could be allocated to comparability. A higher score represented better quality of the study methodology. The maximum score was 4 for selection dimension, 2 for comparability, and 3 for outcome/exposure. A study with a score equal to or higher than 6 was considered of high quality.

2.3. Statistical Analysis. Statistical analysis was performed using RevMan (version 5.3; Cochrane Collaboration, Oxford, United Kingdom) and STATA statistical software (version 12.0; StataCorp, College Station, TX, USA). Data that could not be obtained were to be calculated when necessary. For example, when standard deviation (SD) was not available, it was calculated using the sample sizes and standard error. For continuous outcome, means and standard deviations were used to calculate the pooled weighted mean differences (WMDs) and their 95\% confidence intervals (CIs). The chi-square test, $\operatorname{tau}^{2}$, and the Higgins $I^{2}$ test were used to assess heterogeneity [12]. The $I^{2}$ test is a method for quantifying inconsistency across studies and describes the percentage of variability in effect estimates due to heterogeneity. A value greater than $50 \%$ was considered substantial heterogeneity. A fixed-effects model was adopted for the analysis if the $P$ value was $>0.1$ and the $I^{2}$ index was $<50 \%$, as these results would indicate no between-study heterogeneity. Otherwise, a randomeffects model was applied [13].

Potential publication bias was examined using funnel plots [14]. To evaluate the influence of each individual study on the stability of the overall pooled estimate, we conducted sensitivity analyses by removing each study and observing whether the result changed. Metaregression analysis and subgroup analyses were conducted according to the sample size, publication year, age, sex ratio, and mean PD duration of the cases to explore the potential inherent heterogeneity across the included studies.

\section{Results}

A total of 214 articles were initially identified, and 20 casecontrol studies which met our inclusion criteria were included in the final meta-analysis (Figure 1; Table 1) 


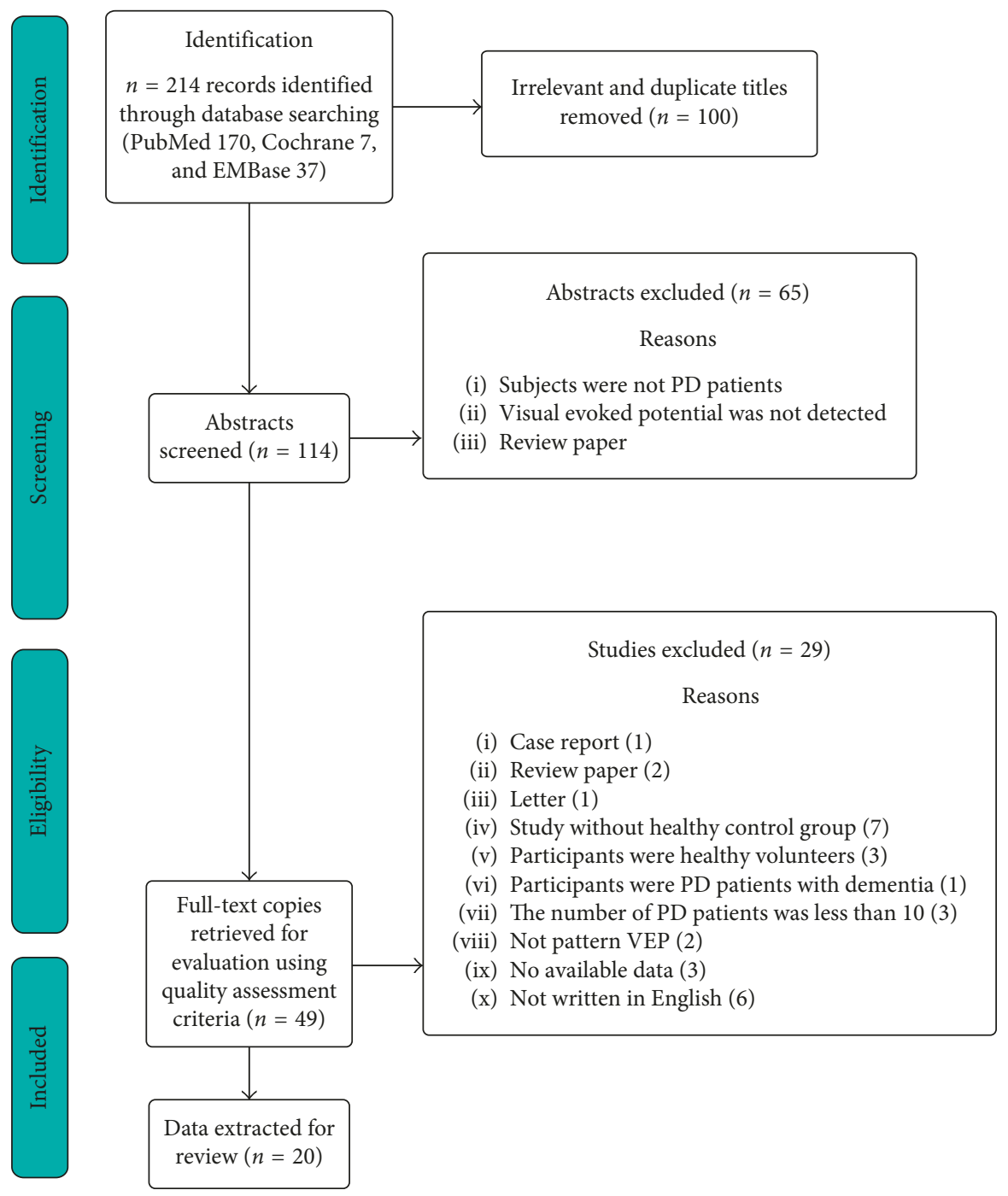

FIgURE 1: Search and study selection process.

[2, 3, 7-10, 15-28]. Seven out of the 20 studies had the NOS score equal to or more than 6 , and the mean score was 6.3.

Because of significant heterogeneity $\left(I^{2}=93 \%\right)$ across the included studies, we used the random-effects model to calculate the WMDs. The P100 latency (WMD $=6.04,95 \%$ CI: 2.73 to $9.35, P=0.0003, n=20$ ), but not amplitude of $\mathrm{P} 100$, was significantly higher in PD patients compared with healthy controls (Figures 2(a) and 2(b)).

Metaregression analysis and subgroup analyses revealed that different sample sizes, sex ratios, and disease durations across studies (all $P<0.05$ ), but not publication year and age (both $P>0.05$ ), were possible sources of heterogeneity observed in the current meta-analysis (Table 2). The difference in P100 latency between PD patients and controls was more pronounced in the studies with a large proportion of men and long PD duration patients $(P$-difference $<0.05)$ than the studies with a small proportion of men and short PD duration patients $(P$-difference $>0.05)$ (Table 2$)$.

Sensitivity analyses showed no significant changes in the pooled WMD or $95 \%$ CI upon the exclusion of any study, indicating that the overall pooled estimates were stable.
The funnel plot did not support existence of publication bias (Figure 3).

\section{Discussion}

In this meta-analysis based on 20 case-control studies, we observed that there is a significant delay in the VEP, as assessed by $\mathrm{P} 100$ latency, in individuals with $\mathrm{PD}$, relative to controls. PD is a disorder of the motor system in which there is no obvious clinical involvement of the visual system and with no pathological lesions (e.g., demyelination), that are considered major determinants of the delays in conduction in visual pathways [2]. Our findings could lead to a better understanding of the physiology of human and primate vision: how and where dopamine acts in the elementary retinal processing of signals related to spatial contrast.

The changes in VEP reflect functional damage in the visual pathway of PD patients. Someone also found structural damage in the retina by using optical coherence tomography which revealed thinning of the retinal nerve fiber layer and macula [29] in PD. The deficit is most evident in 


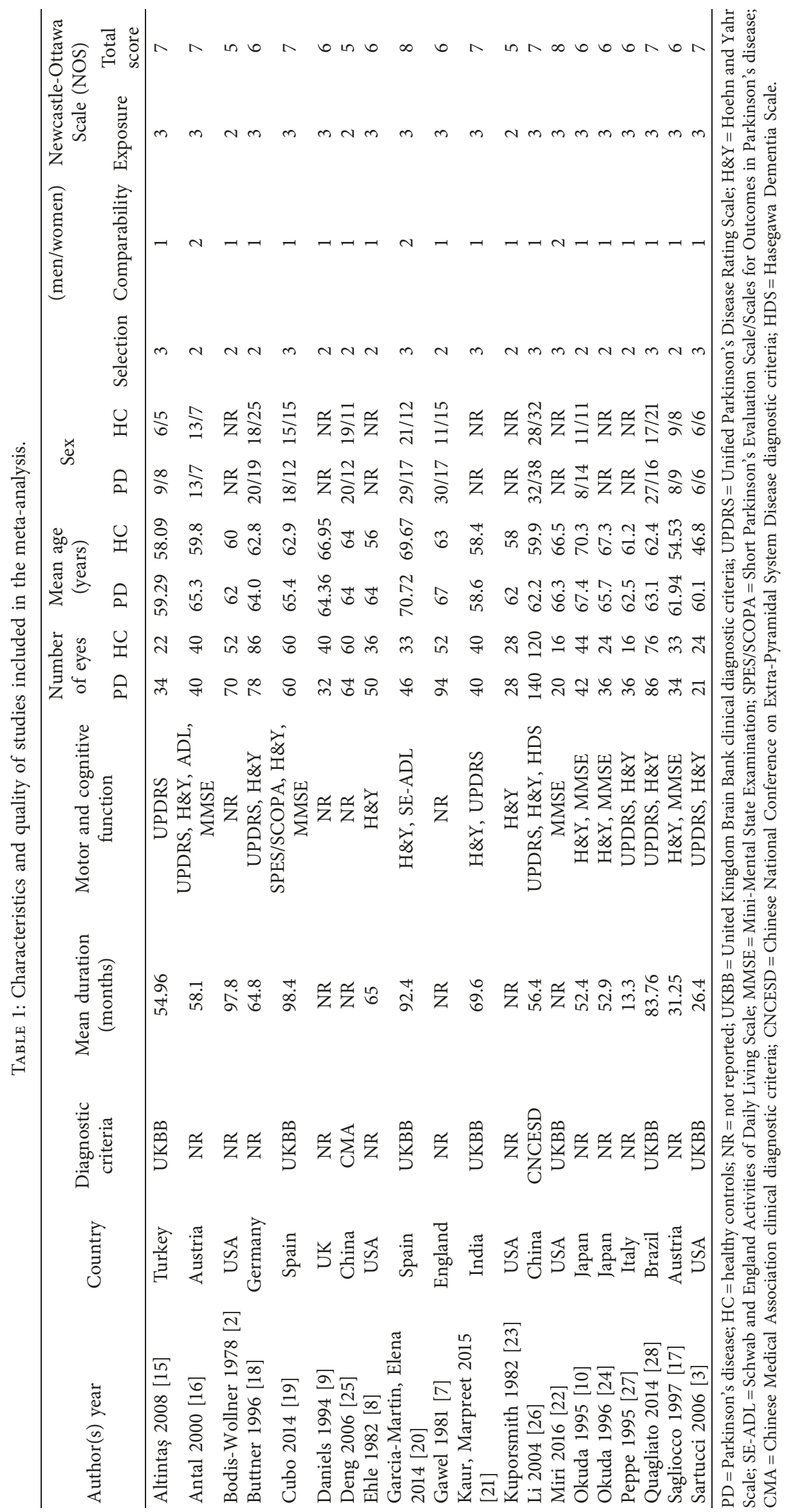




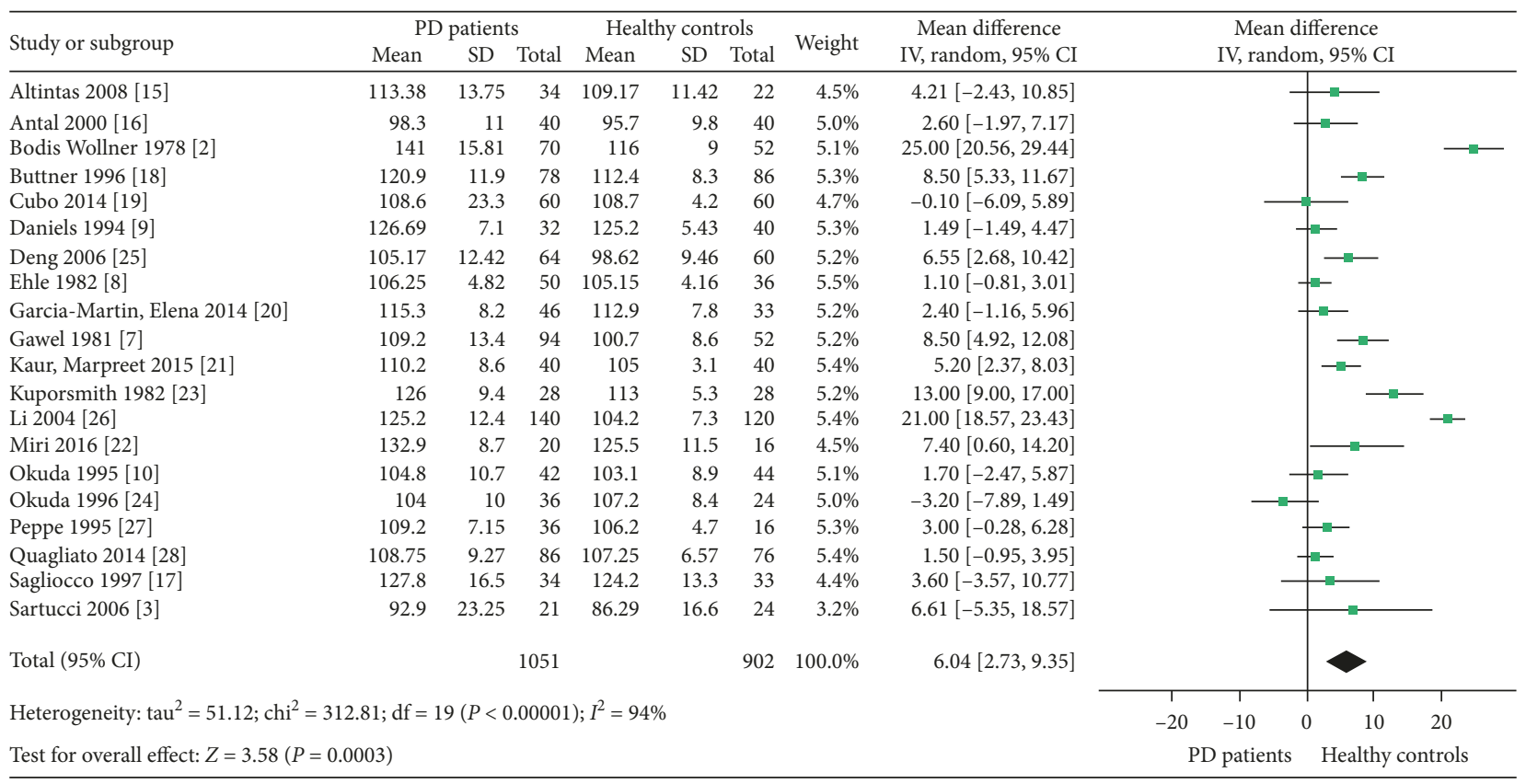

(a)

\begin{tabular}{|c|c|c|c|c|c|c|c|c|c|c|c|c|}
\hline \multirow{3}{*}{$\frac{\text { Study or subgroup }}{\text { Antal } 2000[16]}$} & \multicolumn{3}{|c|}{ PD patients } & \multicolumn{3}{|c|}{ Healthy controls } & \multirow{3}{*}{$\begin{array}{r}\text { Weight } \\
10.4 \%\end{array}$} & \multirow{3}{*}{$\begin{array}{c}\text { Mean difference } \\
\text { IV, random, 95\% CI }\end{array}$} & \multirow{2}{*}{\multicolumn{4}{|c|}{$\begin{array}{c}\text { Mean difference } \\
\text { IV, random, } 95 \% \text { CI }\end{array}$}} \\
\hline & \multirow{2}{*}{$\begin{array}{r}\text { Mean } \\
7.1\end{array}$} & \multicolumn{2}{|c|}{ SD Total } & \multirow{2}{*}{ Mean } & \multirow{2}{*}{$\begin{array}{l}\mathrm{SD} \\
1.6\end{array}$} & \multirow{2}{*}{$\begin{array}{r}\text { Total } \\
40\end{array}$} & & & & & & \\
\hline & & 4 & 40 & & & & & & & & - & \\
\hline Cubo 2014 [19] & 6.4 & 3.3 & 60 & 6.5 & 2.9 & 60 & $11.8 \%$ & $-0.10[-1.21,1.01]$ & & - & 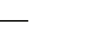 & \\
\hline Daniels 1994 [9] & 7.39 & 3.71 & 32 & 6.35 & 4.1 & 40 & $7.9 \%$ & $1.04[-0.77,2.85]$ & & & $\longrightarrow$ & \\
\hline Ehle 1982 [8] & 7.95 & 4.41 & 50 & 5.9 & 3.27 & 36 & $8.8 \%$ & $2.05[0.43,3.67]$ & & & $\longrightarrow$ & \\
\hline Garcia-Martin, Elena 2014 [20] & 11.9 & 4.5 & 46 & 12.5 & 4.4 & 33 & $7.1 \%$ & $-0.60[-2.59,1.39]$ & & & - & \\
\hline Kaur, Marpreet 2015 [21] & 9.4 & 2.3 & 40 & 8.3 & 1.6 & 40 & $13.4 \%$ & $1.10[0.23,1.97]$ & & & - & \\
\hline Okuda 1995 [10] & 7.5 & 4.1 & 42 & 8.4 & 4 & 44 & $8.3 \%$ & $-0.90[-2.61,0.81]$ & & & - & \\
\hline Peppe 1995 [27] & 5.09 & 2.45 & 36 & 5.74 & 1.35 & 16 & $12.3 \%$ & $-0.65[-1.69,0.39]$ & & & 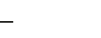 & \\
\hline Sagliocco 1997 [17] & 4.1 & 2.5 & 34 & 3.4 & 1.6 & 33 & $12.5 \%$ & $0.70[-0.30,1.70]$ & & & - & \\
\hline Sartucci 2006 [3] & 8.35 & 3.8 & 21 & 5.02 & 2.56 & 24 & $7.4 \%$ & $3.33[1.41,5.25]$ & & & 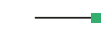 & \\
\hline Total $(95 \% \mathrm{CI})$ & & & 401 & & & 366 & $100.0 \%$ & $0.64[-0.06,1.33]$ & & & & \\
\hline Heterogeneity: $\operatorname{tau}^{2}=0.74 ; \mathrm{chi}^{2}=$ & $\mathrm{df}=9(P$ & $0.004)$ & $I^{2}=$ & & & & & & $\begin{array}{c}1 \\
-4\end{array}$ & $\begin{array}{c}1 \\
-2\end{array}$ & $\begin{array}{l}1 \\
2\end{array}$ & $\begin{array}{l}1 \\
4\end{array}$ \\
\hline Test for overall effect: $Z=1.80(F$ & & & & & & & & & $\mathrm{PD}$ & patients & Healthy $c$ & controls \\
\hline
\end{tabular}

(b)

Figure 2: Pooled weighted mean differences in visual evoked potential: (a) P100 latency and (b) P100 amplitude.

TABLE 2: Metaregression analysis and subgroup analysis.

\begin{tabular}{|c|c|c|c|c|}
\hline Subgroup factor & Assign criteria & Number of studies & WMD (95\% CI) & $P$-difference between groups* \\
\hline \multirow{2}{*}{ Sample size } & $\leq 20$ & 10 & $4.20(1.38,7.02)$ & \multirow{2}{*}{0.001} \\
\hline & $>20$ & 10 & $7.64(2.21,13.1)$ & \\
\hline \multirow{2}{*}{ Publication year } & Before 2000 & 10 & $6.25(1.91,10.6)$ & \multirow{2}{*}{0.12} \\
\hline & 2000 or later & 10 & $5.79(0.49,11.1)$ & \\
\hline \multirow{2}{*}{ Age (years) } & $<64$ & 9 & $9.39(3.04,15.7)$ & \multirow{2}{*}{0.08} \\
\hline & $\geq 64$ & 11 & $3.38(1.18,5.58)$ & \\
\hline \multirow{2}{*}{ Proportion of men } & $>50 \%$ & 8 & $4.47(2.03,6.91)$ & \multirow{2}{*}{0.03} \\
\hline & $\leq 50 \%$ & 4 & $8.44(-3.98,20.9)$ & \\
\hline \multirow{2}{*}{ Duration (months) } & $\leq 56.4$ & 8 & $4.82(-2.31,12.0)$ & \multirow{2}{*}{0.01} \\
\hline & $>56.4$ & 7 & $6.38(1.08,11.6)$ & \\
\hline
\end{tabular}

${ }^{*}$ Adjusted for all factors in this table.

the annular zone surrounding the fovea, where anatomical studies [30] demonstrated the highest concentration of dopaminergic amacrine cells. Dopaminergic deficiency is related to a VEP delay. The postmortem study of PD's retina observed a decrease in retinal dopamine concentration [31]. The thinned inner retina [32] suggests involvement of the 


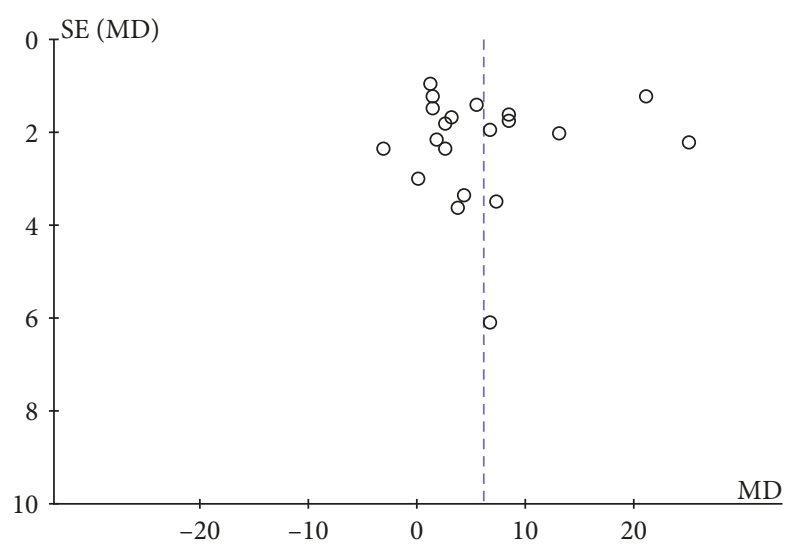

(a)

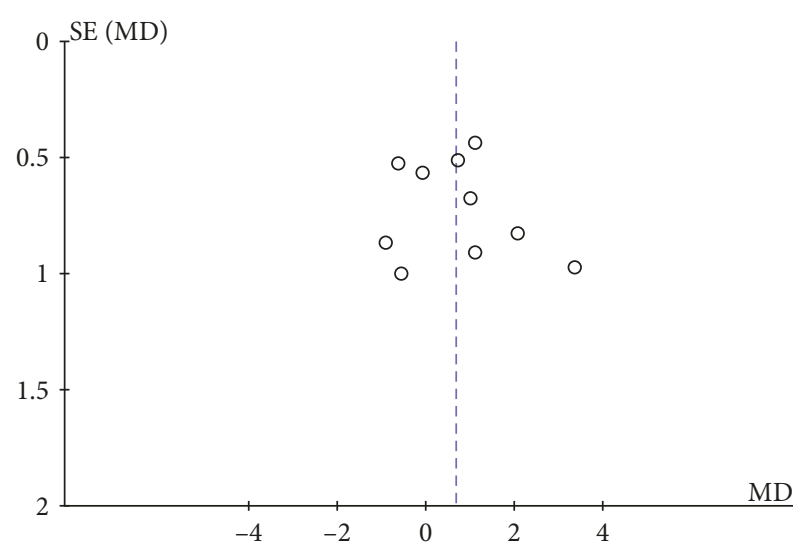

(b)

FIGURE 3: Funnel plots for evaluating the publication bias for visual evoked potential: (a) P100 latency and (b) P100 amplitude.

ganglion cell layer and dopaminergic neurons and the inner plexiform layer in PD. Importantly, previous studies suggested that the structural changes correlated significantly with functional changes $[15,19,21,22]$.

We found a significant delay in the average P100 latency in PD patients compared with the healthy controls, and this difference could be modified by different sample sizes, PD durations, and sex ratios across studies. Disease duration and sex difference may be associated with electrophysiologic changes in the visual pathway of patients with PD. Previous studies reported that the functional changes correlated significantly with disease duration and severity of PD $[21,22]$. Furthermore, a prolonged mean VEP latency in PD patients was shown to be dependent on the sex of the participants (more evident in men than women) [33]. The difference in the thickness of the skull and the brain volume between men and women could also explain, at least in part, the gender difference in VEP for PD patients.

The mechanism of visual defect in PD may act as follows: pathological changes in PD are not only found in the nigrastriatum system but also in the caudal nuclei, putamen nuclei, hypothalamus, and pontine nucleus ceruleus [30]. This could result in cholinergic system dysfunction that diffusely affects the brainstem auditory pathway. The injuries to these regions cause dopaminergic neuron degeneration and decrease in dopamine production and secretion, which may affect the function of the interplexiform cells and horizontal cells in the retina to undermine the transmission of the visual signals, contributing to the abnormal changes of VEP [18]. Then, dopamine exists in the lateral geniculate body and visual cortex, and the visual cortex also contains acetylcholine and its receptors [34]. Long-term dopamine deficiency could trigger a compensatory decrement of acetylcholine and lead to changes in the VEP latency. A retinal dopaminergic deficiency could also underlie some visual changes in PD. However, the relationship between the decrement of dopamine in the nigra-striatum of PD patients and dopamine dysfunction in the visual pathway needs further study. Alpha-synuclein ( $\alpha$-syn) is also expressed widely in the vertebrate retina including humans [35], and it is likely that loss or dysfunction of $\alpha$-syn at this site is responsible for visual symptoms [36].

It is worth noting that VEP measures the integrity of the entire visual pathway. The changes in these potentials in PD may reflect the widespread nature of the biochemical disorder affecting both the retina and central nervous system. Thus, the VEP is a relatively poor method for assessing the anterior visual pathway dysfunction because it is incapable of differentiating impaired macular function from impaired ganglion cell function. Although all the patients were normal on ophthalmological examination, we cannot exclude involvement of the direct visual pathway in PD as a cause of delayed VEP. The pattern electroretinogram (PERG) measures the electrical activity generated by neural and nonneuronal cells in the retina in response to a light stimulus and reflects functional changes in the retina. The PERG would differentiate whether the retinal abnormalities were the cause of the VEP changes. As we expected, some studies reported impaired PERG in PD patients and confirmed the retinal functional defect $[20,21]$. However, they still cannot exclude the disorder in the upper visual pathway. Furthermore, both PERG and VEP improve with therapy, but there is an apparent difference: levodopa therapy improves PERG abnormalities to a higher degree than it does VEP deficits $[27,37,38]$. One possible interpretation is that VEP changes in PD represent secondary nondopaminergic, and therefore more chronic, alterations in visual processing. It seems that additional pathology beyond the retina affects visual responses, including VEPs. Although the role of retinal dysfunction seems certain, the contribution of cortical and lateral geniculate impairment to these visual symptoms remains unknown.

This meta-analysis has some limitations, which are as follows: (1) We did not include unpublished studies and those which were not written in English. The current study thus may lose some high-quality evidences. (2) Because most studies did not provide data regarding PD severity, we failed to explore the potential impacts of disease severity on the VEP-PD relation. (3) We cannot address the potential impact of dopaminergic drugs because most PD cases were 
treated. Previous studies suggested that these treatments could improve contrast sensitivity and reverse VEP delays in $\mathrm{PD}$ patients $[2,39,40]$. Failure to control the use of dopaminergic drugs thus would underestimate the true difference in VEP latency between PD patients and controls. (4) Some participants with other neurodegenerative diseases were probably included in control groups, as screenings are not fully stringent so as to exclude early dementias.

\section{Conclusion}

This meta-analysis showed that the P100 latency was significantly longer in PD patients than healthy controls. The application of VEP provides an approach for a more comprehensive evaluation of the visual pathway and a better understanding of the pathophysiology of visual involvement in PD. However, further researches with longitudinal study design and incorporating the pattern electroretinogram assessment are warranted.

\section{Conflicts of Interest}

The authors declare that there are no conflicts of interest regarding the publication of this article.

\section{Authors' Contributions}

Song-bin $\mathrm{He}$ and Xiang Gao conceived and designed the experiments. Chun-yan Liu, Ya-ping Zhang, and Zhi-nan Ye performed the experiments. Chun-yan Liu, Lin-di Chen, and Bin-da Wang analyzed the data. Wei-guo Tang, Song-bin $\mathrm{He}$, and Xiang Gao contributed reagents/materials/analysis tools. Chun-yan Liu, Lin-di Chen, and Xiang Gao wrote the paper. All authors read and approved the final manuscript.

\section{Acknowledgments}

This work was supported by the project of the Department of Health and Family Planning Bureau of Zhoushan City, Zhejiang Province, China (no. 2014A17 to Song-bin He), and the NIH/NINDS R21-NS087235-02 (to Xiang Gao).

\section{Supplementary Materials}

\section{PRISMA Checklist. (Supplementary Materials)}

\section{References}

[1] A. Rossi, K. Berger, H. Chen, D. Leslie, R. B. Mailman, and X. Huang, "Projection of the prevalence of Parkinson's disease in the coming decades: revisited," Movement Disorders, 2017.

[2] I. Bodis-Wollner and M. D. Yahr, "Measurements of visual evoked potentials in Parkinson's disease," Brain, vol. 101, no. 4, pp. 661-671, 1978.

[3] F. Sartucci and V. Porciatti, "Visual-evoked potentials to onset of chromatic red-green and blue-yellow gratings in Parkinson's disease never treated with L-dopa," Journal of Clinical Neurophysiology, vol. 23, no. 5, pp. 431-436, 2006.

[4] M. F. Ghilardi, E. Chung, I. Bodis-Wollner, M. Dvorzniak, A. Glover, and M. Onofrj, "Systemic 1-methyl,4-phenyl,1-23-6-tetrahydropyridine (MPTP) administration decreases retinal dopamine content in primates," Life Sciences, vol. 43, no. 3, pp. 255-262, 1988.

[5] J. D. Elsworth, J. R. Taylor, J. R. Sladek Jr., T. J. Collier, D. E. Redmond Jr., and R. H. Roth, "Striatal dopaminergic correlates of stable parkinsonism and degree of recovery in old-world primates one year after MPTP treatment," Neuroscience, vol. 95, no. 2, p. 399, 2000.

[6] M. F. Ghilardi, M. S. Marx, I. Bodiswollner, C. B. Camras, and A. A. Glover, "The effect of intraocular 6-hydroxydopamine on retinal processing of primates," Annals of Neurology, vol. 25, no. 4, pp. 357-364, 1989.

[7] M. J. Gawel, P. Das, S. Vincent, and F. C. Rose, "Visual and auditory evoked responses in patients with Parkinson's disease," Journal of Neurology Neurosurgery and Psychiatry, vol. 44, no. 3, pp. 227-232, 1981.

[8] A. L. Ehle, R. M. Stewart, N. E. Lellelid, and N. A. Leventhal, "Normal checkerboard pattern reversal evoked potentials in parkinsonism," Electroencephalography and Clinical Neurophysiology, vol. 54, no. 3, pp. 336-338, 1982.

[9] R. Daniels, G. F. Harding, and S. J. Anderson, "Effect of dopamine and acetylcholine on the visual evoked potential," International Journal of Psychophysiology, vol. 16, no. 2-3, pp. 251-261, 1994.

[10] B. Okuda, H. Tachibana, K. Kawabata, M. Takeda, and M. Sugita, "Visual evoked potentials (VEPs) in Parkinson's disease: correlation of pattern VEPs abnormality with dementia," Alzheimer Disease and Associated Disorders, vol. 9, no. 2, pp. 68-72, 1995.

[11] A. Liberati, D. G. Altman, J. Tetzlaff et al., "The PRISMA statement for reporting systematic reviews and meta-analyses of studies that evaluate healthcare interventions: explanation and elaboration," Epidemiology Biostatistics and Public Health, vol. 6, no. 4, pp. e1-e34, 2009.

[12] A. V. Margulis, M. Pladevall, N. Riera-Guardia et al., "Quality assessment of observational studies in a drug-safety systematic review, comparison of two tools: the Newcastle-Ottawa Scale and the RTI item bank," Clinical Epidemiology, vol. 6, no. 8, p. 359, 2014.

[13] J. P. T. Higgins and S. G. Thompson, "Quantifying heterogeneity in a meta-analysis," Statistics in Medicine, vol. 21, no. 11, pp. 1539-1558, 2010.

[14] J. P. Vandenbroucke, "Bias in meta-analysis detected by a simple, graphical test,” BMJ, vol. 315, no. 7109, pp. 629-634, 1997.

[15] Ö. Altintaş, P. Işeri, B. Özkan, and Y. Cağlar, "Correlation between retinal morphological and functional findings and clinical severity in Parkinson's disease," Documenta Ophthalmologica, vol. 116, no. 2, pp. 137-146, 2008.

[16] A. Antal, G. Dibó, S. Kéri et al., "P300 component of visual event-related potentials distinguishes patients with idiopathic Parkinson's disease from patients with essential tremor," Journal of Neural Transmission, vol. 107, no. 7, pp. 787-797, 2000.

[17] L. Sagliocco, F. Bandini, M. Pierantozzi et al., "Electrophysiological evidence for visuocognitive dysfunction in younger non Caucasian patients with Parkinson's disease," Journal of Neural Transmission, vol. 104, no. 4-5, pp. 427-439, 1997.

[18] T. Buttner, W. Kuhn, T. Muller, T. Heinze, C. Puhl, and H. Przuntek, "Chromatic and achromatic visual evoked potentials in Parkinson's disease," Electroencephalography and Clinical Neurophysiology, vol. 100, no. 5, p. 443, 1996.

[19] E. Cubo, M. J. López Peña, E. Diez-Feijo Varela et al., "Lack of association of morphologic and functional retinal changes with motor and non-motor symptoms severity in Parkinson's 
disease," Journal of Neural Transmission, vol. 121, no. 2, pp. 139-145, 2014.

[20] E. Garcia-Martin, D. Rodriguez-Mena, M. Satue et al., "Electrophysiology and optical coherence tomography to evaluate Parkinson disease severity," Investigative Ophthalmology and Visual Science, vol. 55, no. 2, p. 696, 2014.

[21] M. Kaur, R. Saxena, D. Singh, M. Behari, P. Sharma, and V. Menon, "Correlation between structural and functional retinal changes in Parkinson disease," Journal of neuroophthalmology: the official journal of the North American Neuro-Ophthalmology Society, vol. 35, no. 3, p. 254, 2015.

[22] S. Miri, S. Glazman, L. Mylin, and I. Bodis-Wollner, "A combination of retinal morphology and visual electrophysiology testing increases diagnostic yield in Parkinson's disease," Parkinsonism and Related Disorders, vol. 1, no. 5, pp. 134-137, 2016.

[23] M. J. Kupersmith, E. Shakin, I. M. Siegel, and A. Lieberman, "Visual system abnormalities in patients with Parkinson's disease," Archives of Neurology, vol. 39, no. 5, pp. 284-286, 1982.

[24] B. Okuda, H. Tachibana, M. Takeda, K. Kawabata, and M. Sugita, "Visual and somatosensory evoked potentials in Parkinson's and Binswanger's disease," Dementia, vol. 7, no. 1, pp. 53-58, 1996.

[25] Q. Deng, J. Deng, Y. Zhao, X. Yan, and P. Chen, "Analysis of brain-stem auditory evoked potential and visual evoked potential in patients with Parkinson disease," Neural Regeneration Research, vol. 1, no. 5, pp. 449-452, 2006.

[26] F. Li, D. X. Gu, and Y. J. Li, "Correlation of brainstem auditory evoked potential and visual evoked potential with disturbance of intelligence in patients with Parkinson disease," Chinese Journal of Clinical Rehabilitation, vol. 8, pp. 7002-7004, 2004.

[27] A. Peppe, P. Stanzione, F. Pierelli, A. D. De, M. Pierantozzi, and G Bernardi, "Visual alterations in de novo Parkinson's disease: pattern electroretinogram latencies are more delayed and more reversible by levodopa than are visual evoked potentials," Neurology, vol. 45, no. 6, pp. 1144-1148, 1995.

[28] L. B. Quagliato, C. Domingues, E. M. Quagliato, E. B. Abreu, and N. Karajunior, "Applications of visual evoked potentials and Fourier-domain optical coherence tomography in Parkinson's disease: a controlled study," Arquivos Brasileiros de Oftalmologia, vol. 77, no. 4, pp. 238-242, 2014.

[29] R. Inzelberg, J A. Ramirez, P. Nisipeanu, and A. Ophir, "Retinal nerve fiber layer thinning in Parkinson disease," Vision Research, vol. 44, no. 24, p. 2793, 2004.

[30] C. Savy, A. Simon, and J. Nguyenlegros, "Spatial geometry of the dopamine innervation in the avascular area of the human fovea," Visual Neuroscience, vol. 7, no. 5, pp. 487-498, 1991.

[31] C. Harnois and P. T. Di, "Decreased dopamine in the retinas of patients with Parkinson's disease," Investigative Ophthalmology and Visual Science, vol. 31, no. 11, pp. 2473-2475, 1990.

[32] M. E. Hajee, W. F. March, D. R. Lazzaro et al., "Inner retinal layer thinning in Parkinson disease," Archives of Ophthalmology, vol. 127, no. 6, p. 737, 2009.

[33] S. Nightingale, K. W. Mitchell, and J. W. Howe, "Visual evoked cortical potentials and pattern electroretinograms in Parkinson's disease and control subjects," Journal of Neurology, Neurosurgery, and Psychiatry, vol. 49, no. 11, pp. 1280-1287, 1986.

[34] H. Tachibana, M. Takeda, M. Sugita, J. Kondo, M. Miyauchi, and A. Matsuoka, "Brainstem auditory evoked potentials in patients with multi-infarct dementia and dementia of the
Alzheimer type," International Journal of Neuroscience, vol. 37, no. 2, pp. 325-331, 2009.

[35] G. C. Martíneznavarrete, J. Martínnieto, J. Esteverudd, A. Angulo, and N. Cuenca, "Alpha synuclein gene expression profile in the retina of vertebrates," Molecular Vision, vol. 13, pp. 949-961, 2007.

[36] H. A. Lashuel, C. R. Overk, A. Oueslati, and E. Masliah, "The many faces of $\alpha$-synuclein: from structure and toxicity to therapeutic target," Nature Reviews Neuroscience, vol. 14, no. 1, pp. 38-48, 2013.

[37] P. A. Bhaskar, S. Vanchilingam, E. A. Bhaskar, A. Devaprabhu, and R. Ganesan, "Effect of L-dopa on visual evoked potential in patients with Parkinson's disease," Neurology, vol. 36, no. 8, pp. 1119-1121, 1986.

[38] A. Peppe, P. Stanzione, M. Pierantozzi et al., "Does pattern electroretinogram spatial tuning alteration in Parkinson's disease depend on motor disturbances or retinal dopaminergic loss?," Electroencephalography and Clinical Neurophysiology, vol. 106, no. 4, pp. 374-382, 1998.

[39] L. Barbato, S. Rinalduzzi, M. Laurenti, S. Ruggieri, and N. Accornero, "Color VEPs in Parkinson's disease," Electroencephalography and Clinical Neurophysiology/Evoked Potentials Section, vol. 92, no. 2, pp. 169-172, 1994.

[40] M. Onofrj, M. F. Ghilardi, M. Basciani, and D. Gambi, "Visual evoked potentials in parkinsonism and dopamine blockade reveal a stimulus-dependent dopamine function in humans," Journal of Neurology Neurosurgery, and Psychiatry, vol. 49, no. 10, pp. 1150-1159, 1986. 


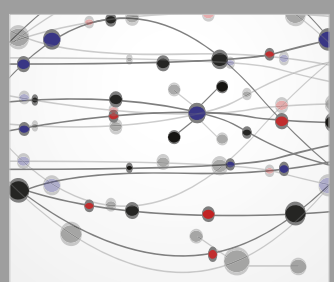

The Scientific World Journal
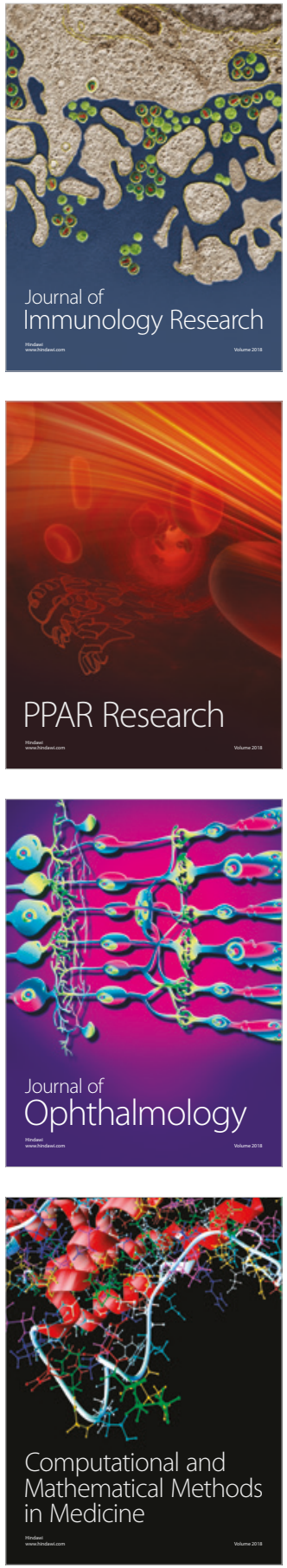

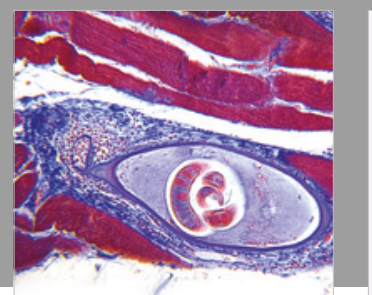

Gastroenterology Research and Practice

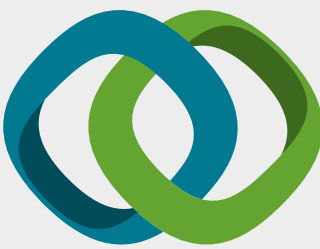

\section{Hindawi}

Submit your manuscripts at

www.hindawi.com
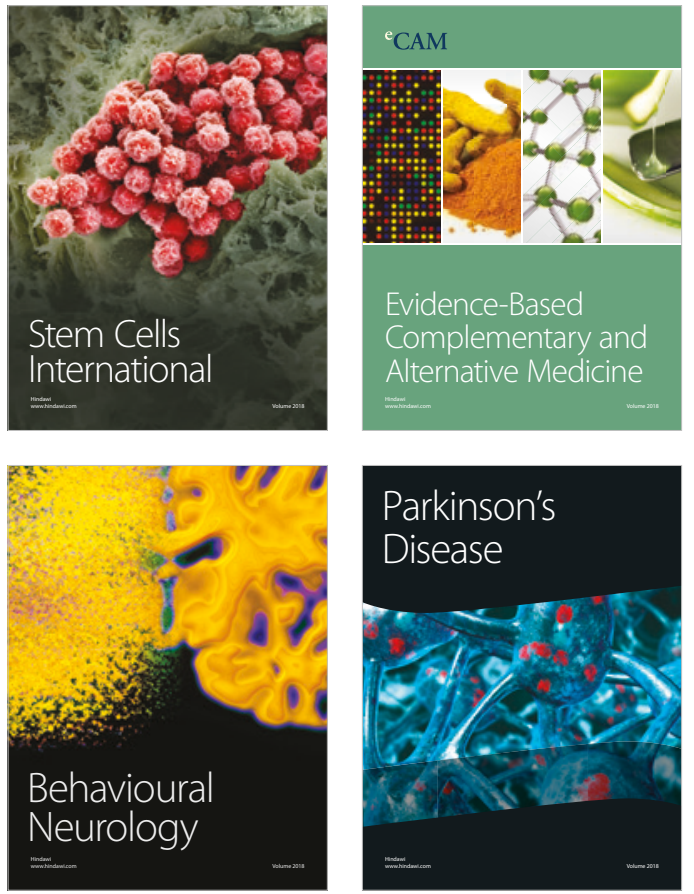

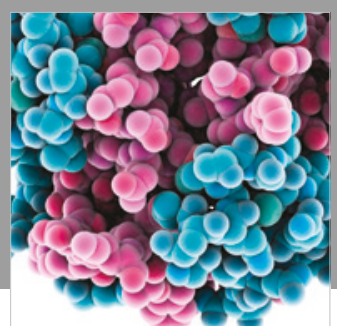

ournal of

Diabetes Research

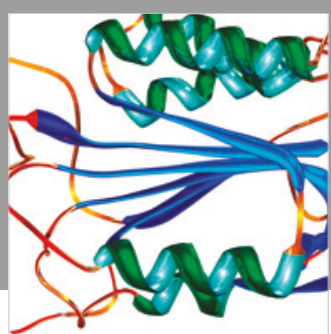

Disease Markers
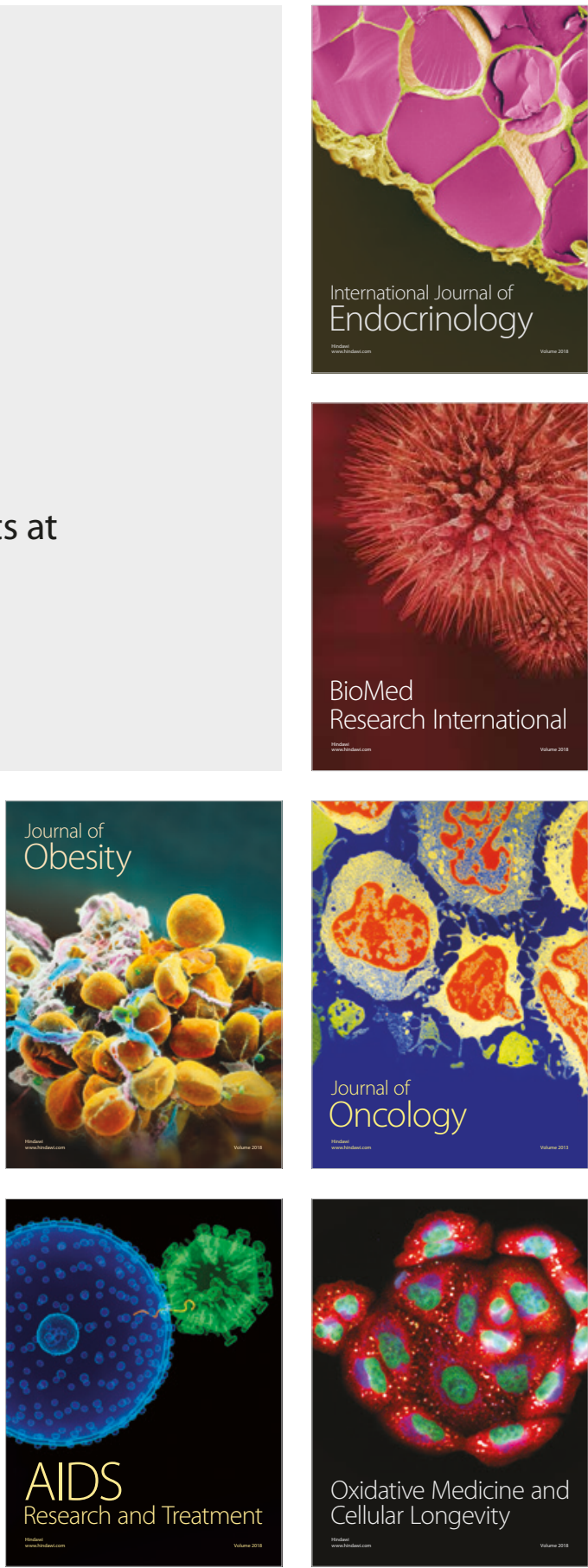